\title{
Manejo de la tecnología por parte de los docentes
}

\author{
María Del Carmen Bordón de Del Puerto \\ maria.bordon@mec.edu.py \\ Maestría en Ciencias de la Educación, con énfasis en Educación \\ Universidad Nacional de Pilar \\ Pilar Paraguay
}

\section{RESUMEN}

El avance del COVID en Paraguay y en el mundo ha llevado al cambio de la rutina en el ámbito educativo, con la suspensión de las clases presenciales evidenciando la necesidad de utilizar la tecnología. En este contexto, esta investigación pretende describir de qué manera incide el manejo de la tecnología por parte de los docentes de la Educación Escolar Básica Región 1 Nivel 1 Cerrito, Laureles y Villalbín en el año 2020, determinar las dificultades y limitaciones del manejo de las TIC, identificar las competencias de los docentes en su uso para su aplicación en el proceso de enseñanza - aprendizaje, citar los avances y mejoras obtenidos en el proceso, identificar el mecanismo de interacción más común utilizados por docentes y alumnos.

La metodología será de tipo descriptivo, cuantitativo y de corte transversal, comprende la descripción, registro, análisis e interpretación de los datos obtenidos, además el diseño y aplicación del instrumento para obtener los resultados, confrontarlos con los basamentos teóricos, información primaria y secundaria, generar las conclusiones y las recomendaciones.

Se da conocer la realidad en el uso de la tecnología (equipos y plataformas) de los docentes antes y durante de la pandemia, su utilización en el proceso de Enseñanza Aprendizaje, se recopilan experiencias de los docentes, se concluye y se da las recomendaciones.

Palabras clave: manejo; tecnología, plataformas. 


\title{
Technology management by teachers
}

\begin{abstract}
The advance of COVID in Paraguay and in the world has led to a change in the routine in the educational field, with the suspension of face-to-face classes evidencing the need to use technology. In this context, this research aims to describe how the management of technology affects the teachers of the Basic School Education Region 1 Level 1 Cerrito, Laureles and Villalbín in the year 2020, to determine the difficulties and limitations of the management of the ICT, identify the skills of teachers in their use for their application in the teaching - learning process, cite the progress and improvements obtained in the process, identify the most common interaction mechanism used by teachers and students. The methodology will be descriptive, quantitative and cross-sectional, it includes the description, registration, analysis and interpretation of the data obtained, as well as the design and application of the instrument to obtain the results, compare them with the theoretical bases, primary and secondary information, generate conclusions and recommendations.

The reality in the use of technology (equipment and platforms) of teachers before and during the pandemic is made known, its use in the Teaching-Learning process, experiences of teachers are collected, it is concluded and recommendations are given .
\end{abstract}

Keywords: management; technology, platforms.

Artículo recibido: 10. Junio. 2021 Aceptado para publicación: 16. Julio. 2021 Correspondencia: maria.bordon@mec.edu.py

Conflictos de Interés: Ninguna que declarar 


\section{INTRODUCCIÓN}

El avance del COVID en Paraguay y en el mundo ha llevado al cambio de la rutina de los países afectados en menor y mayor escala. También ha generado un cambio en la política de la modalidad de la educación.

La pandemia de enfermedad por coronavirus (COVID-19) ha provocado una crisis sin precedentes en todos los ámbitos. En la esfera de la educación, esta emergencia ha dado lugar al cierre masivo de las actividades presenciales de instituciones educativas en más de 190 países con el fin de evitar la propagación del virus y mitigar su impacto. Según datos de la Organización de las Naciones Unidas para la Educación, la Ciencia y la Cultura (UNESCO), a mediados de mayo de 2020 más de 1.200 millones de estudiantes de todos los niveles de enseñanza, en todo el mundo, habían dejado de tener clases presenciales en la escuela. (UNESCO, 2020)

En el ámbito educativo, gran parte de las medidas que los países de la región han adoptado ante la crisis se relacionan con la suspensión de las clases presenciales en todos los niveles, lo que ha dado origen a tres campos de acción principales: el despliegue de modalidades de aprendizaje a distancia, mediante la utilización de una diversidad de formatos y plataformas (con o sin uso de tecnología); el apoyo y la movilización del personal y las comunidades educativas, y la atención a la salud y el bienestar integral de las y los estudiantes. (UNESCO, 2020)

En el marco de la suspensión de las clases presenciales, la necesidad de mantener la continuidad de los aprendizajes ha impuesto desafíos que los países han abordado mediante diferentes alternativas y soluciones en relación con los calendarios escolares y las formas de implementación del currículo, por medios no presenciales y con diversas formas de adaptación, priorización y ajuste. Para realizar los ajustes se requiere tomar en cuenta las características de los currículos nacionales o subnacionales, los recursos y capacidades del país para generar procesos de educación a distancia, los niveles de segregación y desigualdad educativa del país y el tiempo transcurrido del año escolar. (UNESCO, 2020). El mundo día a día se beneficia de la tecnología que trae una serie de ventajas, sobre todo en el ámbito educativo donde ha revolucionado la forma de enseñar, así como de aprender, hoy más que nunca necesitamos de ella para acceder a la educación en tiempos de pandemia, donde los estudiantes se ven impedidos para acudir a los establecimientos educativos debido a la cuarentena, esto hace que tanto estudiantes como 
docentes busquen formas de superar las brechas digitales para así poder acceder a una educación de calidad y calidez. (Crespo Argudo, M. C - Palaguachi, M.C, 2020)

La pandemia ha transformado los contextos de implementación del currículo, no solo por el uso de plataformas y la necesidad de considerar condiciones diferentes a aquellas para las cuales el currículo fue diseñado, sino también porque existen aprendizajes y competencias que cobran mayor relevancia en el actual contexto. Es preciso tomar una serie de decisiones y contar con recursos que desafían a los sistemas escolares, los centros educativos y los docentes. Tal es el caso de los ajustes y las priorizaciones curriculares y la contextualización necesaria para asegurar la pertinencia de los contenidos a la situación de emergencia que se vive, a partir del consenso entre todos los actores relevantes. Es igualmente importante que en estos ajustes se prioricen las competencias y los valores que se han revelado como prioritarios en la actual coyuntura: la solidaridad, el aprendizaje autónomo, el cuidado propio y de otros, las competencias socioemocionales, la salud y la resiliencia, entre otros.

La investigación se inicia con el Planteamiento y Formulación del problema, en donde se exponen las directrices y los componentes fundamentales del estudio, es decir, toda la problemática que involucra el presente tema de investigación y que nos conduce a la pregunta a la que se pretende dar respuesta, seguido de los objetivos propuestos.

En el marco teórico, se expone toda la documentación, es decir, toda la base teórica clave que sirvió de apoyo para el desarrollo de la investigación. Luego se encuentra el marco metodológico, en donde se exponen de manera lógica y sustentada, el diseño de la investigación acorde al nivel de desarrollo del problema y de los objetivos respectivos.

Seguidamente se realiza un Análisis y Discusión de Resultados, se presentan varios gráficos, producto de los datos obtenidos durante la realización de esta investigación con la finalidad de dar respuesta a los objetivos planteados.

Por último, se presenta las Conclusiones y Recomendaciones a las que se pudo llegar con base a los resultados obtenidos y a los objetivos planteados.

\section{ESTRATEGIAS METODOLÓGICAS O MATERIALES Y MÉTODOS}

La investigación se realiza en la zona de Supervisión Pedagógica Región 1 Nivel 1 de las localidades de Cerrito, Laureles y Villalbín del Departamento de Neembucú, con 65 docentes de aulas de la Educación Escolar Básica. 
La metodología utilizada es de tipo descriptivo, comprende la descripción, registro, análisis e interpretación de la naturaleza actual, y la composición o proceso de los fenómenos.

El enfoque se hace sobre conclusiones dominantes o sobre grupo de personas, grupo o cosas, se conduce o funciona en presente (Tamayo y Tamayo, 2003). De enfoque cuantitativo, el cual parte de una idea que va acotándose y, una vez delimitada, se derivan objetivos y preguntas de investigación, se revisa la literatura y se construye un marco o una perspectiva teórica. De corte transversal que analiza datos de variables recopiladas en un periodo de tiempo sobre una población muestra o subconjunto predefinido (Hernández Sampieri, Fernández Collado, \& Baptista Lucio, 2014).

Para la recolección de los datos en el presente trabajo de investigación se utilizaron las siguientes fuentes:

- Fuentes Primarias: Las fuentes primarias de la investigación se encuentran relacionada fundamentalmente con los docentes de la mencionada zona, que constituyen la fuente de primera mano y son los principales protagonistas de la investigación

- Fuentes Secundarias: Estas fuentes constituyen las informaciones escritas recopiladas de los libros, diccionarios y revistas, que sustentan al mismo tiempo la teoría del trabajo de investigación realizado.

La población está compuesta por 65 docentes de aulas de 29 instituciones de la zona mencionada de Supervisión Educativa, en los turnos mañana y tarde, de la Escolar Básica Nivel 1 Región 1 Neembucú. La muestra es la misma cantidad de docentes de aulas, atendiendo que es una cantidad que se puede investigar de manera total, es decir $100 \%$. El Muestreo, los docentes que forman el universo y que por lo tanto están incluidos en la muestra, pues, está al alcance del investigador.

\section{Métodos y Técnicas de Recolección de Datos}

En primer lugar, se solicita el permiso correspondiente en la Dirección para realizar la presente investigación.

Las técnicas empleadas para la recogida de la muestra, y dada la situación que se está atravesando por el brote del COVID-19, se decide realizar mediante un cuestionario Google Forms (https://www.google.com/forms/about) que se difunde mediante plataformas virtuales para los docentes de aula. Una vez analizada la base de datos en el 
programa Microsoft Excel (https://products.office.com/), se realiza un análisis de los ítems de respuesta.

\section{Validación de expertos}

Para llevar a cabo este proceso, se construye un formato de validación, el cual incluye las instrucciones sobre las cuales deben guiar a los jueces expertos y emitir su opinión, para realizarle las modificaciones pertinentes, garantizando que el instrumento mida los aspectos de estudio, al respecto Hernández y otros (2006), indican que "se refiere al grado en que un instrumento realmente mide la variable que pretende medir" (p.277).

Es decir, se relaciona la coherencia entre el instrumento y lo que se desea medir. En cuanto a la confiabilidad, Hernández y otros (2006) dicen que "se refiere al grado en que su aplicación repetida al mismo sujeto u objeto produce resultados iguales. (p.277) Asimismo, se constata si el instrumento contiene el índice apropiado de manejo y dominio para medir las dimensiones y las variables del estudio. Se aplica a 65 docentes de aulas. Como resultado de la encuesta se obtiene la certeza en cuanto a la validez y la confiabilidad de los instrumentos.

\section{Procedimientos}

a) El procedimiento para la realización de la investigación objeto de estudio, es a través de:

b) Selección de la situación problemática, con la formulación de los objetivos justificación y delimitación

c) Solicitud de permiso a los actores educativos y la Dirección

d) Elección de los antecedentes de la investigación y revisión de la bibliografía.

e) Determinación de la metodología a seguir en la investigación; es decir el tipo y nivel de la investigación, diseño y población objeto de estudio.

f) Finalmente, se incluye además el diseño del instrumento de obtención de datos, fuentes de información primaria y documentos como fuentes de información secundaria, así como la validez, confiabilidad, y el plan de análisis estadístico de los datos, aplicación de los cuestionarios para obtener los resultados, confrontarlos con los basamentos teóricos y generar las conclusiones y las recomendaciones del estudio.

\section{Plan de análisis e interpretación de los resultados}

Una vez que se recolectan los datos a través de la aplicación del instrumento, se procede a analizar la información obtenida que permite determinar el logro de los objetivos 
planteados al inicio de la investigación. La presentación y análisis de los resultados para los objetivos de la investigación, se realiza a través del uso del método de estadística descriptiva, utilizando las técnicas de medidas de tendencia central y de variabilidad. En este sentido, Hernández y otros (2006), alegan que consiste en asignar a cada unidad a una o más categorías". (p.364).

\section{RESULTADOS Y DISCUSIÓN}

La práctica docente en los Distritos de Villalbín, Laurales y Cerrito ha tenido un rotundo impacto durante el estricto confinamiento a causa del covid 19, en cuanto a toda la práctica docente y el proceso de enseñanza -aprendizaje, en cuestión de días el docente necesitó rever todas sus prácticas. 42 docentes señalaron que ha tenido un alto impacto, mientras que 22 docentes mencionaron un mediano impacto, ninguna de ellas mencionó haber tenido un bajo impacto, esta situación crítica no fue indiferente para nadie.

En una escala del 1 al 5, los docentes han calificado el impacto de esta situación en el proceso de enseñanza - aprendizaje en un promedio de 3,37, específicamente 45 docentes han mencionado que ha sido difícil adaptarse al nuevo rol, mientras que 5 docentes mencionaron que les resultó muy difícil adaptarse a la nueva manera de trabajar, asimismo a 9 docentes les pareció fácil y para 6 docentes sin novedad.

En cuanto a los equipos tecnológicos que los docentes utilizaban con regularidad antes de la pandemia se encuentra en primer lugar el celular, pues 53 docentes, equivalentes al $81 \%$ manifestaron utilizarlo con regularidad, mientras que otros equipos informáticos solo 16 docentes, equivalentes al $25 \%$, una importante cantidad de docentes, equivalente a un $12 \%$ no utilizaba ningún tipo de aparatos tecnológicos y tampoco una Tablet antes de la pandemia.

Se puede evidenciar que todos los docentes tuvieron que esforzarse para aprender a utilizar aparatos tecnológicos o herramientas de dichos aparatos que no conocían con anterioridad. Tal es así que 41 docentes debieron aprender a usar su celular para el desarrollo de sus clases, ya sea para enviar tareas, realizar explicaciones o enviar pequeños videos explicativos de la clase, mientras que 32 personas debieron aprender a utilizar computadoras o equipos informáticos, una persona Tablet y solo 9 docentes ya utilizaban algún tipo de equipos con anterioridad.

Cabe destacar que durante trabajos realizados en la zona se ha detectado docentes que se esforzaron para comprar equipos informáticos para el desarrollo de sus clases y/o archivo 
de tareas y trabajos, exponiendo sus compras con mucho orgullo, mientras que en años anterior lo veían muy lejano o casi imposible poderlos manejar.

En absoluta mayoría los docentes de la zona mencionada desarrollaron clases a través del WhatsApp para la interacción con sus alumnos, pues es el recurso disponible y que mejor manejan, en una ínfima cantidad se ha mencionado utilizar otros recursos, pues un solo docente manifestó utilizar My Teams, otro Meet Google y otro Zoom. Y 3 manifestaron otras como las fotocopias. Muchos docentes no tienen la menor idea de cómo utilizar otras plataformas; incluso My Teams, un recurso que el Ministerio de Educación y Ciencia facilitó y promocionó la utilización para las clases virtuales encuentra mucha resistencia en los docentes.

La mayor dificultad o limitaciones con que han tenido que lidiar durante el proceso de enseñanza- aprendizaje los docentes, es el acceso a internet, lastimosamente en todo el departamento de Ñeembucú y por qué no decirlo en todo Paraguay, no se cuenta con una efectiva señal de internet. Seguido de carencia de celulares en los hogares, 31 docentes han manifestado tropezar con esta dificultad, en muchos casos un solo celular para varios alumnos de diferentes grados en mismo hogar; 29 docentes manifestaron que otra dificultad no menor con la que debieron lidiar fue la saturación de los teléfonos celulares en los hogares, atendiendo que muy pocas familias cuentan con un móvil de alta gama; la economía familiar es otro factor que incidió enormemente para el desarrollo de las clases y con ello la falta de saldo; 22 docentes manifestaron esta dificultad, mientras que 17 docentes manifestaron que la falta de equipos informáticos fue otra dificultad; también la falta de fotocopias o la barrera para conseguirlo, en la opción de otras se presentó en un menor cantidad. Cabe resaltar que, a pesar de toda esta serie de dificultades que debieron sortear, los docentes siguieron y sostuvieron la educación en tiempos de pandemia.

Los docenes reconocieron que es de suma importancia el manejo de la tecnología para el desarrollo de las clases y hacer uso de los recursos virtuales que ofrecen las diferentes plataformas a fin de compartir, proyectar, grabar sus clases, un total de 51 docentes señalaron esta realidad, 22 docentes afirmaron que se requiere de conocimientos, 21 docentes manifestaron que se necesita habilidades y destrezas, mientras que 2 docentes señalaron otras como cobertura y/o acceso a internet. Podemos concluir sin ninguna duda, que las competencias de los docentes en el uso de la tecnología eran muy escasas y sigue siendo escasa, aunque se debe reconocer que se ha dado un paso gigantesco y están en el 
proceso de adoptarla definitivamente como manera de trabajar en un futuro muy cercano, y que hará el trabajo sea más sencillo; como todo cambio llevara su tiempo para lograrlo. Con relación a la pregunta de cuál era la experiencia en cuanto a las evaluaciones online, las respuestas giraron en torno a: muy interesante, significativa, enriquecedora, muy buena, difícil, con dificultad, es más fácil el procesamiento. Lo más utilizado fue a través de Form, atendiendo que los alumnos podían responder desde el celular, aparato más utilizado para el desarrollo de clases y evaluaciones.

Los docentes se van adaptando de a poquito a esta nueva manera de trabajar, la experiencia es positiva, cada vez más van experimentando y dependiendo de la tecnología, por lo que en adelante se irá utilizando con mayor frecuencia, los niños tienen más facilidad para enfrentar una educación diferente, o que ayudará en este proceso, será muy útil para la retroalimentación de actividades curriculares y extracurriculares, Ayudará a los niños a construir sus propios aprendizajes más rápido, práctico y más independientes, mucho más útil será en la formación continua del Docente en servicio. Ayudará a crear una generación emprendedora con uso de la tecnología y su aplicación también en todos los campos laborales.

La confianza que los docentes han expresado sentir al emplear los medios tecnológicos en el proceso de enseñanza- aprendizaje es de un promedio de 3,54. Aunque también reconocen que el proceso en sí de Inicio, desarrollo y cierre solo a veces han podido cumplir cabalmente. Cabe resaltar que esa confianza expresada es solo con relación a los aparatos tecnológicos que ellos manejan bien, es decir el celular, por lo que tienen pensado seguir utilizando aquello que conoce más o sabe utilizar más, por lo que 63 docentes manifestaron que utilizarán el WhatsApp, 9 señalaron que utilizarían Zoom, 7 Meet Google y otras 5 y 4 respectivamente My Teams y correo electrónico.

Absoluta mayoría reconoce que necesita una capacitación para conocer el manejo y las herramientas que tienen las diferentes plataformas. Se puede concluir que los docentes usaron y siguen usando solamente las herramientas que conocen y están muy abiertos para aprender y usar otras, lo que podría facilitarles el trabajo, toda vez que los gobiernos a través de los organismos gubernamentales proporcionen una buena señal de internet y las herramientas necesarias para un programa exitoso de clases a distancia. 


\section{CONCLUSIÓN O CONSIDERACIONES FINALES}

La práctica docente en los Distritos de Villalbín, Laurales y Cerrito ha tenido un rotundo impacto durante el estricto confinamiento a causa del covid 19, en cuanto a toda la práctica docente y el proceso de enseñanza -aprendizaje, en cuestión de días el docente necesitó rever todas sus prácticas, pasando de clases presenciales a clases virtuales o a distancia. El equipo tecnológico más utilizado por los mismos fue el celular y la herramienta más utilizada el WhatsApp. Todos los docentes tuvieron que esforzarse para aprender a utilizar aparatos tecnológicos o herramientas de dichos aparatos que no conocían con anterioridad, muchos de ellos hicieron un esfuerzo económico para comprar un equipo informático, necesario para bajar los materiales proveídos por el Ministerio de Educación y Ciencias a través de Portal digital, para guardar las tareas remitiditas por sus alumnos y realizar todo el trabajo que conlleva una educación a distancia. Este proceso fue realmente difícil y en una escala de 1 al 5, según la percepción de los docentes el impacto sería de 3,37

La mayor dificultad con lo que se ha tropezado es el acceso a internet. Es necesario que el gobierno a través de los entes gubernamentales proporcione una buena señal de internet; de suma utilidad a toda la comunidad educativa en todo el país, para a poyar a la educación a distancia. Seguido de carencia de celulares en los hogares, en muchos casos un solo celular para varios alumnos de diferentes grados en mismo hogar, otra dificultad no menor con la que debieron lidiar fue la saturación de los teléfonos celulares en los hogares, atendiendo que muy pocas familias cuentan con un móvil de alta gama, la economía familiar es otro factor que incidió enormemente para el desarrollo de las clases y con ello la falta de saldo, la falta de equipos informáticos fue otra dificultad así como la falta de fotocopias.

A pesar de que este proceso fue difícil, se puede destacar también algunas ventajas tales como: mayor rapidez, innovación en cuanto al uso tecnológico, los alumnos estudian desde la comodidad de sus casas, facilidad para el acceso de la información, menor riesgo de contagio de virus, no se corta la educación y da mayor participación a los padres en el proceso enseñanza de sus hijos, amplias posibilidades formativas, fomento de la autonomía.

Se ha evidenciado a través de esta investigación que los docentes tienen muy poco conocimiento, habilidades y destrezas, en general el manejo de la tecnología, por lo que 
urge una capacitación para el mejor aprovechamiento de todas las herramientas disponibles.

Se puede concluir que este confinamiento obligó a dar un paso importante y en el futuro ayudará a los niños a construir sus propios aprendizajes más rápido, práctico y más independientes, será fundamental en la formación continua del Docente en servicio. Ayudará a crear una generación emprendedora con uso de la tecnología y su aplicación en todos los campos laborales.

Finalmente, no podemos hablar de retroceso en la educación, pero sí una manera diferente de concebir la manera de enseñar y de aprender, pues la experiencia de la educación a distancia tiene sus ventajas y sus desventajas, tal como se describo más arriba. El resultado de esta investigación será utilizado para ejecutar un proyecto de capacitación de docente en la zona, sobre utilización de las diferentes plataformas digitales y sus herramientas, para el mejoramiento del proceso de enseñanza- aprendizaje, y lograr una educación a distancia de calidad.

\section{REFERENCIAS BIBLIOGRAFICAS}

Barbera, E. y Badia, A. (2005). "Hacia el Aula Virtual: actividades de enseñanza y aprendizaje en la red". Revista Iberoamericana de Educación. Vol $36 N^{\circ}$ 9, 1-21.

Britez, M. (2020). La educación ante el avance del COVID-19 en Paraguay. Comparativo con países de la Triple Frontera. ScieloPreprints.

Campa, R. (31 de Setiembre de 2020). Educación, Política y Valores. Revista Dilemas Contemporáneos, 1(VIII).

Crespo Argudo, M. C - Palaguachi, M.C. (2020). Educación con Tecnología en una Pandemia: Breve Análisis. Revista Scientific, 5(17).

Cueva, J.., García, A., \& Martínez, O. (2019). El conectivismo y las TIC: Un paradigma que impacta el proceso enseñanza aprendizaje. Revista Scientific, 205-227.

Delgado, P. (Julio de 2020). La enseñanza de las matemáticas requiere una urgente reestructuración, señala nuevo reporte. https://observatorio.tec.mx/edunews/ensenanza-de-las-matematicas-covid19.

García, et al. (s/d). Las Tic en el aprendizaje. Salamanca España.

Gómez Dávalos, N R y Rodriguez Fernández, P. (2020). ESTRÉS EN DOCENTES EN EL CONTEXTO DE LA PANDEMIA DE COVID-19 Y LA EDUCACION, 
FENOB UNA - FILIAL CORONEL OVIEDO. Academic Disclosure UNA FENOB, 216-234.

Leyes Paraguayas, L. 6. (17 de junio de 2020). http://www.bacn.gov.py/leyesparaguayas. Obtenido de http://www.bacn.gov.py

Lizaraso, F., \& Del Carmen, J. (2020). Coronavirus y las amenazas a la salud mundial. Obtenido de https://doi.org: https://doi.org/10.24265/horizmed

Lozano Vargas, A. (2020). Impacto de la epidemia del Coronavirus (COVID-19) en la salud mental del personal de salud y en la población general de China. Revista de NeuroPsiquiatria [revista en Internet].

Marín, N. (2019). Las Tecnologías de Información y Comunicación: Una Gestión Educativa desde la Plataforma Moodle. Revista Scientific.

Martínez, O. (2018a,b). Uso de las Tecnologías de la Información y la Comunicación en la Educación Básica. Revista Scientific, 154-174.

Moya, A. (2009). Las nuevas tecnologías en Educación.

Osorio, M. (2012). Unidad 1: Globalización, Tecnología, Sociedad del Conocimiento y Tecnologías de la Información y la Comunicación (TIC): Aproximaciones para situar el binomio educación-tecnología en el Copyright SENA @, 2012. Bogotá.

Pinedo, I. (2017). Programa de Capacitación Docente: Tecnologías del Aprendizaje y del Conocimiento - TACs. Panamá: Universidad Especializada de las Américas.

Piña-Ferrer, L. (2020). El COVID 19: Impacto psicológico en los seres humanos. Rev Arbitr Interdiscip Ciencias la Salud Salud y Vida, 188.

Quintero-Corzo, J., Munévar-Molina, R., \& Munévar-Quintero, F. . (2015). Nuevas tecnologías, nuevas enfermedades en los entornos educativos. Hacia la promoción de la Salud.

Ramírez Vega, A., \& Chacón Rivas, M. (2011). Math Bridge: una propuesta como apoyo en los procesos de enseñanza-aprendizaje de las matemáticas en Costa Rica (CO). XIII CONFERÊNCIA INTERAMERICANA DE EDUCAÇÃO MATEMÁTICA. Costa Rica.

Sánchez, G., \& Montenegro, A. (2019). Teletrabajo una propuesta de innovación en productividad empresarial. 
Tacca Huamán, D. R., \& Tacca Huamán, A. L. (2019). Factores de riesgos psicosociales y estrés percibido en docentes universitarios. Propósitos y Representaciones. Obtenido de https://doi.org: https://doi.org/10.20511/pyr2019.v7n3.304

UNESCO. (2020). La educación en tiempos del coronavirus.

UNESCO, C. (2020). La educación en tiempos de la Pandemia de COVID 19.

Zuñiga Vega, C. (2004). “Aula Virtual: nueva herramienta para la educación ambiental. Revista Biocenosis, $N^{o}$ 18, Vol.1, 38-42. 